\title{
RUDJER BOŠKOVIĆ INSTITUTE RADIOCARBON MEASUREMENTS XVII
}

\author{
Nada Horvatinčić ${ }^{1}$ Ines Krajcar Bronić • Bogomil Obelić • Jadranka Barešić \\ Rudjer Bošković Institute, P.O. Box 180, 10002 Zagreb, Croatia.
}

ABSTRACT. This paper presents dating results of geological (speleothems, tufa, soil, and sediment), biological (mollusks and botanical), as well as hydrogeological samples from Croatia, Slovenia, Bosnia and Herzegovina, Turkey, and China. Included are results of samples measured by gas proportional counting (GPC) in the Zagreb lab until abandonment of this technique in 2007, as well as results of several series measured by both GPC and liquid scintillation counting (LSC) methods.

\section{INTRODUCTION}

This report presents dating of different geological samples, mainly carbonate deposits as speleothems (submerged and terrestrial), tufa, and lake sediments, as well as hydrogeological samples. We also present here some environmental samples such as plants, soil, and shells. Samples were measured by gas proportional counting (GPC) prior to 2007 and since 2003 by liquid scintillation counting (LSC) using sample preparation in the form of methane (Srdoč et al. 1971, 1979) and either benzene or direct absorption of $\mathrm{CO}_{2}$ (Horvatinčić et al. 2004), respectively. We use oxalic acid I and oxalic acid II as modern standards for GPC and LSC measurements, respectively.

${ }^{14} \mathrm{C}$ results are presented as the ratio of radiocarbon activities, $\mathrm{a}^{14} \mathrm{C}$, in percent of modern carbon (pMC) and their ages are given as conventional ${ }^{14} \mathrm{C}$ ages. Age calculations follow the conventional protocol (Mook and van der Plicht 1999) based on the Libby half-life of $5570 \pm 30 \mathrm{yr}$ and using AD 1950 as the reference year. Ages and standard deviations ( $1 \sigma$ error) of samples are adjusted for stable isotope fractionation to the normalized concentration ratio $\left(\delta^{13} \mathrm{C}=-25 \%\right)$ according to the recommendations in Stuiver and Polach (1977) and using the default $\delta^{13} \mathrm{C}$ values if not measured. Whenever available, measured $\delta^{13} \mathrm{C}$ and $\delta^{18} \mathrm{O}$ values are also given. Calibrated ages of some organic samples are calculated from non-rounded ${ }^{14} \mathrm{C}$ conventional ages by using OxCal v 4.1 (Bronk Ramsey 2009, 2012) and the IntCal09 data (Reimer et al. 2009) with $1 \sigma$ error (confidence level 68.2\%).

It should be noted here that for carbonate sediments such as speleothems, tufa, lake sediments, as well as for water samples, the conventional ${ }^{14} \mathrm{C}$ age is not the real age of these materials. To determine the true/real age, one should take into account the initial ${ }^{14} \mathrm{C}$ activity (or dead carbon proportion, or reservoir effect). A comprehensive study of isotopic and geochemical characteristics of carbonate sediments has been performed in the continental karst areas of Croatia (Krajcar Bronić et al. 1992; Horvatinčić et al. 2003). It was found that the initial ${ }^{14} \mathrm{C}$ activity $\mathrm{a}_{0}$ varies between 65 and $90 \mathrm{pMC}$, depending on local conditions. However, data presented in this paper are not corrected for the reservoir effect.

A new relational database for ${ }^{14} \mathrm{C}$ samples has been recently developed (Portner et al. 2009). The quality assurance and quality control system according to ISO 17025 has been improved within the IAEA TC Regional Project on Quality Control and Quality Assurance for Nuclear Analytical Techniques. The laboratory participated in ${ }^{14} \mathrm{C}$ intercomparison studies (Horvatinčić et al. 1990; Krajcar Bronić et al. 1995; Sironić et al. 2012). The detection limits for GPC measurements are between 0.5 and $0.7 \mathrm{pMC}$, depending on the stability of the system in a certain period, and $0.4 \mathrm{pMC}$ for LSC measurements (Krajcar Bronić et al. 2009).

\footnotetext{
${ }^{1}$ Corresponding author. Email: nada.horvatincic@irb.hr.
} 


\section{GEOLOGICAL SAMPLES}

\section{Submerged Speleothems in the Adriatic Sea}

\section{Croatia}

In order to reconstruct the Late Pleistocene-Holocene sea-level rise along the eastern Adriatic coast of Croatia, speleothems were collected from submarine caves along the coast (Figure 1). Speleothems are typically continental structures, precipitated only in subareal conditions. These were subsequently submerged and covered by marine biogenic overgrowth. Such speleothems with overgrowth provide datable material for reconstructing sea-level changes.

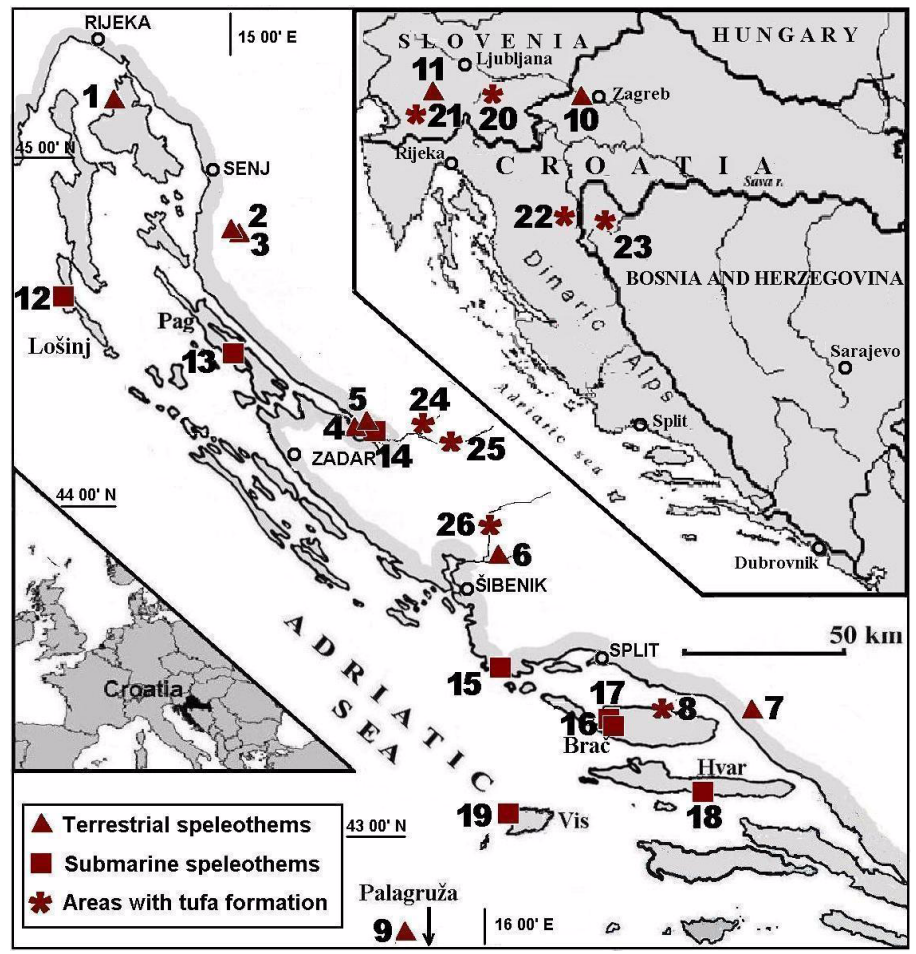

Figure 1 Locations of terrestrial and submarine caves and areas of tufa deposits along the Adriatic coast of Croatia (middle) and locations in Slovenia, inland Croatia, and Bosnia and Herzegovina (top right).

\section{Brač, Pag, and Rogoznica Series}

Submerged speleothems were collected from 3 caves from seawater depths of -38.5 to $-17 \mathrm{~m}: 1$ )

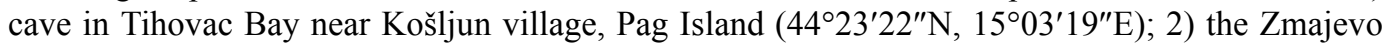
Uho pit near Rogoznica $\left(43^{\circ} 31^{\prime} 57^{\prime \prime} \mathrm{N}, 1^{\circ} 57^{\prime} 44^{\prime \prime} \mathrm{E}\right)$; and 3) a pit in Lučica Bay near Milna, Brač Island ( $\left.43^{\circ} 18^{\prime} 11^{\prime \prime} \mathrm{N}, 16^{\circ} 27^{\prime} 14^{\prime \prime} \mathrm{E}\right)$ (Figure 1, nr 13, 15, and 16, respectively; Table 1). Samples were collected by divers T Rađa and P Tasić, Croatian Scuba Diving Federation, and submitted in 2000 by M Surić, Faculty of Philosophy, University of Zadar. The marine biogenic overgrowth layer that occasionally penetrates speleothems (sample type $\mathrm{p}$ in Figure 2 and Table 1), the youngest (sample type a), and the oldest parts (sample type b) of the speleothems were separated and dated. Their sta- 
ble isotope content, $\delta^{13} \mathrm{C}$ and $\delta^{18} \mathrm{O}$, was also measured (Surić et al. 2005a,b). $\delta^{13} \mathrm{C}$ values indicated different origins of carbon in speleothem samples a and b (precipitation in karst terrestrial environment) and marine overgrowth $\mathrm{p}$ (precipitation in seawater). The aim of investigations was to assess sea-level oscillations.

Table $1{ }^{14} \mathrm{C}$ activities and conventional ages and $\delta^{13} \mathrm{C}$ and $\delta^{18} \mathrm{O}$ values of submerged speleothems of the Brač, Pag, and Rogoznica series. Notations a, b, and p correspond to the speleothem layers described in the Figure 2 caption below.

\begin{tabular}{|c|c|c|c|c|c|c|}
\hline Sample code & Sample & $\begin{array}{l}\text { Sea depth } \\
(\mathrm{m})\end{array}$ & $\begin{array}{l}\mathrm{a}^{14} \mathrm{C} \\
(\mathrm{pMC})\end{array}$ & $\begin{array}{l}\delta^{13} \mathrm{C} \\
(\% \text { PDB })\end{array}$ & $\begin{array}{l}\delta^{18} \mathrm{O} \\
(\% \text { PDB })\end{array}$ & $\begin{array}{l}\text { Age } \\
(\mathrm{BP})\end{array}$ \\
\hline \multicolumn{7}{|c|}{ Brač Island series } \\
\hline Z-3030 & B-38-p & 38.5 & $49.1 \pm 0.8$ & -2.6 & -1.4 & $5710 \pm 130$ \\
\hline Z-3032 & B-38-a & & $2.7 \pm 0.5$ & -7.4 & -4.2 & $28,900 \pm 1600$ \\
\hline Z-3033 & B-38-b & & $<0.5$ & -9.5 & -4.9 & - \\
\hline Z-3035 & B-36-p & 36.0 & $56.9 \pm 0.9$ & -2.3 & -1.7 & $4530 \pm 120$ \\
\hline Z-3036 & B-36-a & & $3.8 \pm 0.5$ & -8.7 & -4.7 & $26,300 \pm 1060$ \\
\hline Z-3037 & B-36-b & & $1.0 \pm 0.5$ & -9.0 & -5.1 & $37,300 \pm 5000$ \\
\hline Z-3038 & B-34-p & 34.0 & $59.5 \pm 0.9$ & -1.8 & -1.0 & $4170 \pm 120$ \\
\hline Z-3039 & B-34-a & & $8.5 \pm 0.6$ & -8.8 & -5.4 & $19,800 \pm 540$ \\
\hline Z-3040 & B-34-b & & $<0.5$ & -9.7 & -5.8 & - \\
\hline Z-3041 & B-28-p & 28.0 & $69.6 \pm 0.7$ & 0.5 & 0.2 & $2910 \pm 80$ \\
\hline Z-3042 & B-28-a & & $8.9 \pm 0.6$ & -7.2 & -4.0 & $19,450 \pm 530$ \\
\hline Z-3060 & B-26-p & 26.0 & $82.0 \pm 1.0$ & 2.5 & 1.7 & $1590 \pm 95$ \\
\hline \multicolumn{7}{|c|}{ Pag Island series } \\
\hline Z-3053 & P-23-p & 23.0 & $63.1 \pm 0.9$ & 1.8 & 1.9 & $3700 \pm 110$ \\
\hline Z-3054 & P-23-a & & $3.6 \pm 0.5$ & -7.5 & -4.7 & $26,800 \pm 1230$ \\
\hline Z-3055 & P-23-b & & $2.1 \pm 0.5$ & -8.5 & -5.3 & $31,030 \pm 2110$ \\
\hline \multicolumn{7}{|c|}{ Rogoznica series } \\
\hline Z-3056 & $\mathrm{R}-21-\mathrm{p}$ & 21.4 & $76.2 \pm 1.0$ & 0.3 & 0.3 & $2180 \pm 100$ \\
\hline Z-3057 & $\mathrm{R}-21-\mathrm{a}$ & & $5.0 \pm 0.5$ & -6.8 & -4.1 & $24,100 \pm 890$ \\
\hline Z-3058 & $\mathrm{R}-21-\mathrm{b}$ & & $2.4 \pm 0.4$ & -6.2 & -4.9 & $29,830 \pm 1300$ \\
\hline Z-3059 & $\mathrm{R}-17-\mathrm{p}$ & 17.0 & $81.3 \pm 1.0$ & 1.4 & 1.0 & $1660 \pm 95$ \\
\hline
\end{tabular}

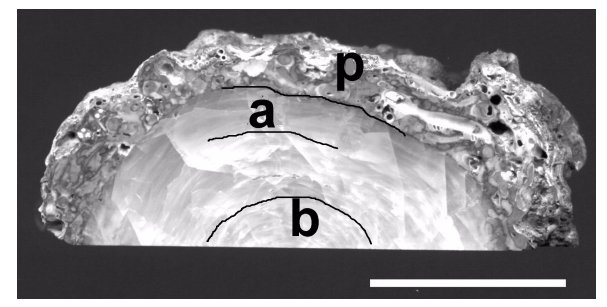

Figure 2 Speleothem layers: $a=$ the youngest; $b=$ the oldest; $p=$ marine biogenic overgrowth.

\section{Vrulja Zečica Series}

Submerged stalagmites from the submarine spring Vrulja Zečica $\left(44^{\circ} 15^{\prime} 54^{\prime \prime} \mathrm{N}, 15^{\circ} 31^{\prime} 43^{\prime \prime} \mathrm{E}\right)$ near Rovanjska, Zadar County, Dalmatia (Figure 1, nr 14). Collected in July 1999 by M Kuhta (Z-2857), Croatian Geological Survey, Zagreb, and September 2003 by M Surić (Z-3660) as part of an investigation to assess of sea-level oscillations. 
Z-2857 Vrulja Zečica, Z-41

$11,150 \pm 160$

$\delta^{13} \mathrm{C}=-10.3 \%, \delta^{18} \mathrm{O}=-4.9 \%$

$25.0 \pm 0.5 \mathrm{pMC}$

Z-3660 Vrulja Zečica, Z-41-S

$10,950 \pm 170$

$\delta^{13} \mathrm{C}=-10.3 \%$, $\delta^{18} \mathrm{O}=-4.9 \%$

$25.5 \pm 0.5 \mathrm{pMC}$

Submerged stalagmite $\sim 40 \mathrm{~cm}$ long at $\sim 42 \mathrm{~m}$ seawater depth; 5-mm layer scraped off from surface

(Surić et al. 2004).

\section{Špurina Cave Series}

Stalactite from the submarine cave Šupurina, west of Komiža, Vis Island, Dalmatia $\left(43^{\circ} 02^{\prime} 40^{\prime \prime} \mathrm{N}\right.$, $16^{\circ} 03^{\prime} 50^{\prime \prime}$ E; Figure 1, nr 19). Collected and submitted in September 1998 by B Jalžić, Croatian Museum of Natural History, Zagreb. Water depth $16 \mathrm{~m}$, upper $2 \mathrm{~m}$ freshwater, below to the bottom seawater; water temperature $19^{\circ} \mathrm{C}$. Layers separated by V Bermanec, Geology Department, Faculty of Natural Science, Zagreb.

$Z-2849$ Inner layer of stalactite
$\delta^{13} \mathrm{C}=-11.1 \%, \delta^{18} \mathrm{O}=-6.2 \%$

Z-2850 Middle layer of stalactite

$\delta^{13} \mathrm{C}=-9.8 \%$ o, $\delta^{18} \mathrm{O}=-5.9 \%$ o

Z-2851 Surface layer of stalactite

$\delta^{13} \mathrm{C}=-8.4 \%, \delta^{18} \mathrm{O}=-5.8 \%$ o

Z-2743 Živa Voda Cave

$\delta^{13} \mathrm{C}=-7.2 \%, \delta^{18} \mathrm{O}=-4.8 \%$

Stalactite from the submarine cave Živa Voda near Bogomolje-Zaglav village in Kožja Bay, Hvar

Island $\left(43^{\circ} 07^{\prime} 01^{\prime \prime} \mathrm{N}, 17^{\circ} 02^{\prime} 52^{\prime \prime} \mathrm{E}\right.$; Figure $\left.1, \mathrm{nr} 18\right)$. Collected and submitted in July 1997 by T Rađa.

\section{Bijaka Cave Series}

Speleothem from the submarine cave Bijaka (Batista jama) near Milna, Brač Island $\left(43^{\circ} 19^{\prime} \mathrm{N}\right.$, $16^{\circ} 26^{\prime} \mathrm{E}$; Figure 1, nr 17). Collected in February 1997 by T Rađa during an investigation of sea-level oscillations.

\section{Z-2717 Bijaka Cave 1}

$$
13,750 \pm 380
$$

Inner, crystal part of the speleothem.

\section{Z-2718 Bijaka Cave 2}

$3990 \pm 160$

Surface part of the speleothem

\section{Medvjeđa Spilja Cave Series}

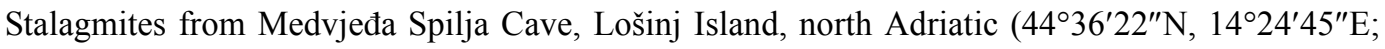
Figure 1, nr 12) described in Surić et al. (2007). The cave is partly submerged by seawater. Collected in April 2004 by M Surić. Samples taken from the submerged part of the cave during an investigation of sea-level oscillations (Surić and Juračić 2010).

\section{Z-3495 Medvjeđa Spilja L-1-S}

$4460 \pm 120$

Stalagmite at $1 \mathrm{~m}$ water depth, surface part.

$57.4 \pm 0.9 \mathrm{pMC}$ 
Stalagmite at $10 \mathrm{~m}$ water depth, surface part.

\section{Speleothems from Terrestrial Caves}

Croatia

\section{Modrič Špilja Series}

Stalagmite from Modrič Špilja Cave, $35 \mathrm{~m}$ asl, near Rovanjska, Zadar County $\left(44^{\circ} 15^{\prime} 25^{\prime \prime} \mathrm{N}\right.$, $15^{\circ} 32^{\prime} 14^{\prime \prime}$ E; Figure 1, nr 5). Collected in July 1999 by M Kuhta (Z-2859) and September 2003 by M Surić (Z-3659).

Z-2859 Modrič Špilja stalagmite

Base of stalagmite $2 \mathrm{~m}$ high.

\section{Z-3659 Modrič Špilja, MOD-5A-215}

$36,100 \pm 2300$

Stalagmite, surface layer; same stalagmite as above.

Stalagmite from a cave found during construction of Maslenica Bridge, north of Zadar $\left(44^{\circ} 14^{\prime} 13^{\prime \prime} \mathrm{N}\right.$, $15^{\circ} 31^{\prime} 20^{\prime \prime}$ E; Figure 1, nr 4). No vegetation was found above cave ceiling. Submitted in April 1994 by D Janičić, Institute for Civil Engineering, Zagreb, to establish the time of neotectonic movements.

\section{Z-2510 Omišalj}

$<0.6 \mathrm{pMC}$

Speleothem in the cave Omišalj, Krk Island $\left(45^{\circ} 12^{\prime} 44^{\prime \prime} \mathrm{N}, 14^{\circ} 32^{\prime} 36^{\prime \prime} \mathrm{E}\right.$; Figure 1, nr 1). Submitted in April 1994 by D Janičić. No vegetation was found above cave ceiling. Dated to establish the time of neotectonic movements.

\section{Z-2841 Palagruža}

Stalactite from the cave at the eastern part of Vela Palagruža Island south Adriatic Sea $\left(42^{\circ} 23^{\prime} 37^{\prime \prime} \mathrm{N}\right.$, $16^{\circ} 14^{\prime} 59^{\prime \prime}$; Figure 1, nr 9). Submitted in January 1999 by D Lacković, Croatian Museum of Natural History, Zagreb (sample from Museum collection, nr 600:ZAG;9231:MP1), in an effort to establish the geologic history of the island. Depth of cave ceiling 2-3 m below plant cover.

\section{Ledena Jama Pit Series}

Speleothem and wood from Ledena Jama pit (depth $536 \mathrm{~m}$ ) in Lomska Duliba Valley $\left(44^{\circ} 46^{\prime} 16^{\prime \prime} \mathrm{N}\right.$, $15^{\circ} 01^{\prime} 34^{\prime \prime} \mathrm{E} ; 1235 \mathrm{~m}$ asl; Velebit Mountain, Figure 1, nr 2). Ice deposit found in the entrance of pit, $\sim 40 \mathrm{~m}$ thick and $\sim 15 \mathrm{~m}$ in diameter. Samples collected and submitted in July 1995 by V Božić, Croatian Speleological Society (Horvatinčić and Božić 2001; Jelinić et al. 2001).

\section{Z-2562 Wood 1}

$150 \pm 100$

$98.2 \pm 1.2 \mathrm{pMC}$

Wooden branch found in ice deposit $\sim 15$ m below ice surface; cal AD 1690-1730 (17.1\%), 1800$1930(50.3 \%)$. 
Wooden branch at the bottom of ice deposit, $40 \mathrm{~m}$ below ice surface; cal AD 1680-1740 (19.9\%), $1800-1930$ (48.3\%).

Z-2598 Wood 3

$175 \pm 85$

$97.8 \pm 1.1 \mathrm{pMC}$

Part of sample Z-2583; cal AD 1650-1700 (14\%), 1720-1820 (30.6\%), 1830-1880 (12.3\%), 1910$1960(11.4 \%)$.

\section{Z-2584 Speleothem}

$31,300 \pm 3600$

Speleothem at $60 \mathrm{~m}$ depth; ${ }^{230} \mathrm{Th} / 234 \mathrm{U}$ age: $301,000 \pm 55,000$.

\section{Slovačka Jama Pit Series}

Speleothems from Slovačka Jama pit (depth 1268 m) in Rožanski Kukovi, Velebit Mountain $\left(44^{\circ} 45^{\prime} 40^{\prime \prime} \mathrm{N}, 15^{\circ} 00^{\prime} 12^{\prime \prime} \mathrm{E}\right.$; $1520 \mathrm{~m}$ asl; Figure 1, nr 3). Collected in October 1996 (Z-2670) and July 1998 (Z-2811 to Z-2813) by D Lacković (Lacković et al. 1999).

\section{Z-2670 Slovačka Jama 1}

$33,500 \pm 2600$

Stalactite from the horizontal relic phreatic passage at $350 \mathrm{~m}$ depth.

Z-2810 Slovačka Jama 2

$33,800 \pm 2500$

$\delta^{13} \mathrm{C}=+5.3 \%, \delta^{18} \mathrm{O}=-6.3 \%$

$1.5 \pm 0.5 \mathrm{pMC}$

Stalactite (SJ/98/6), depth $356 \mathrm{~m}$. Same place as Z-2670.

Z-2811 Slovačka Jama 3

$13,600 \pm 260$

$\delta^{13} \mathrm{C}=+0.9 \%, \delta^{18} \mathrm{O}=-7.1 \%$

$18.3 \pm 0.6 \mathrm{pMC}$

Flowstone from the vadose meander channel (SJ/98/1/3), depth $625 \mathrm{~m}$.

Z-2812 Slovačka Jama 4

$33,300 \pm 2000$

$\delta^{13} \mathrm{C}=+2.1 \%$, $\delta^{18} \mathrm{O}=-6.5 \%$

$1.6 \pm 0.4 \mathrm{pMC}$

Top of stalactite (SJ/98/8), subrecent phreatic channel, depth $1254 \mathrm{~m}$.

\section{Z-2818 Slovačka Jama 5}

$\delta^{13} \mathrm{C}=+2.1 \%$, $\delta^{18} \mathrm{O}=-6.5 \%$

$24,000 \pm 640$

Base of stalactite Z-2812.

$5.0 \pm 0.4 \mathrm{pMC}$

\section{Z-2813 Slovačka Jama 6}

$\delta^{13} \mathrm{C}=+1.1 \%$, $\delta^{18} \mathrm{O}=-7.7 \%$ o

Stalagmite (SJ/98/10), the same location as Z-2812.

$25,700 \pm 700$

$4.1 \pm 0.4 \mathrm{pMC}$

Z-2843 Torak

$7050 \pm 135$

$\delta^{13} \mathrm{C}=-9.9 \%, \delta^{18} \mathrm{O}=-6.4 \%$ o

$41.6 \pm 0.7 \mathrm{pMC}$

Speleothem from Torak Cave in Čikola River Canyon, National Park Krka, Dalmatia $\left(43^{\circ} 49^{\prime} \mathrm{N}\right.$, $16^{\circ} 01^{\prime} \mathrm{E}$; Figure 1, nr 6). Collected in September 1998 by B Jalžić from $9.5 \mathrm{~m}$ water depth.

\section{Veternica Cave Series}

Layer of calcite crystals formed on dolomite rock in Veternica Cave, "Monkey Passage," Medvednica Mountain, Zagreb $\left(45^{\circ} 50^{\prime} \mathrm{N}, 15^{\circ} 52^{\prime} \mathrm{E}\right.$; Figure 1, nr 10). Collected in May 1999 by D Lacković and $\mathrm{N}$ Horvatinčić to study the calcite precipitation at the air-water border (paleolake level). 
Z-2992 V1/1

$<0.4$ pMC

$\delta^{13} \mathrm{C}=-9.6 \%$, $\delta^{18} \mathrm{O}=-7.8 \%$ o

Calcite layer, $\sim 500 \mathrm{~m}$ from cave entrance.

Z-2994 V2/1

$<0.4$ pMC

$\delta^{13} \mathrm{C}=-10.6 \%, \delta^{18} \mathrm{O}=-8.3 \%$ o

Calcite layer, $\sim 50 \mathrm{~m}$ from $\mathrm{V} 1 / 1$ towards cave entrance.

Z-2996 V1/3

$34,100 \pm 3000$

$\delta^{13} \mathrm{C}=-9.6 \%, \delta^{18} \mathrm{O}=-7.9 \%$ 。

$1.4 \pm 0.5 \mathrm{pMC}$

Calcite crystals, $10 \mathrm{~cm}$ below V1/1.

\section{Vilenjača Cave Series}

Stalagmite from the cave Tučepska Vilenjača, which is at the bottom of a 100-m-high rock, with scarce vegetation, Biokovo Mountain, near Makarska, Dalmatia $\left(43^{\circ} 16^{\prime} 06^{\prime \prime} \mathrm{N}, 17^{\circ} 03^{\prime} 51^{\prime \prime} \mathrm{E}\right.$; Figure 1, nr 7). Submitted in April 2002 by D Lacković.

Z-3135 Stalagmite, outer layer

$26,200 \pm 760$

Z-3136 Stalagmite, central part

$3.8 \pm 0.4 \mathrm{pMC}$

$<0.5 \mathrm{pMC}$

Tufa

Croatia

\section{Krka River Series}

Tufa from Bilušića Buk waterfall, broken, dry tufa barrier of Krka River near the village Marasovine, National Park Krka, Dalmatia $\left(44^{\circ} 00^{\prime} 34^{\prime \prime} \mathrm{N}, 16^{\circ} 05^{\prime} 03^{\prime \prime} \mathrm{E}\right.$; Figure 1, nr 26). Collected by D Marguš, National Park Krka, and submitted in May 2000 by B Mihelčić, Rudjer Bošković Institute.

\section{Z-2971 Krka 1}

$2260 \pm 105$ $75.5 \pm 1.0 \mathrm{pMC}$

Brownish, compact tufa well-stratified, middle part of barrier.

\section{Z-2972 Krka 2}

Brownish, soft tufa, surface part of barrier.

\section{Krupa River Series}

Tufa from Krupa River, tributary of Zrmanja River, near Krupa Monastery, north Dalmatia $\left(44^{\circ} 12^{\prime} \mathrm{N}, 15^{\circ} 54^{\prime} \mathrm{E}\right.$; Figure 1, nr 24). Collected and submitted in July 1999 by J Kapelj and G Pavlović, Institute of Geology, Zagreb (Kapelj 2002).

\section{Z-2888 Krupa Zr-4/1}

$5525 \pm 130$

$\delta^{13} \mathrm{C}=-9.5 \%, \delta^{18} \mathrm{O}=-7.6 \%$

Porous, stratified, hard tufa on barrier $\sim 0.5 \mathrm{~m}$ high in water stream.

Hard, compact tufa from the bottom of brook, $1 \mathrm{~m}$ downstream from barrier Zr-4/1. 
Z-2890 Krupa Zr-4/3

$\delta^{13} \mathrm{C}=-9.0 \%, \delta^{18} \mathrm{O}=-8.2 \%$ o

$1155 \pm 95$

Hard, compact tufa from the bottom of the brook.

Z-2891 Krupa Zr-4/4

$1090 \pm 100$

$\delta^{13} \mathrm{C}=-10.5 \%, \delta^{18} \mathrm{O}=-7.6 \%$

$87.3 \pm 1.1 \mathrm{pMC}$

Porous, hard tufa from barrier Z-4/1 with moss overgrowth.

\section{Z-2361 Lovrečina}

Tufa, dry deposit, Lovrečina, near Postire $\left(43^{\circ} 22^{\prime} 17^{\prime \prime} N, 16^{\circ} 39^{\prime} 58^{\prime \prime} \mathrm{E}\right)$, Brač Island. Submitted in 1990 by Lj Marjanac, Institute for Paleontology and Geology of the Quaternary Period, Zagreb.

\section{Zrmanja Series}

Tufa of different texture from Zrmanja River, north Dalmatia, was collected at the surface of 3 tufa barriers/waterfalls in water stream (recent tufa) and out of stream (dry tufa): Berberi Buk $\left(44^{\circ} 11^{\prime} 58^{\prime \prime} \mathrm{N}, 15^{\circ} 45^{\prime} 55^{\prime \prime} \mathrm{E}\right)$; Ogari Buk $\left(44^{\circ} 11^{\prime} 38^{\prime \prime} \mathrm{N}, 15^{\circ} 47^{\prime} 31^{\prime \prime} \mathrm{E}\right)$; and Jankovića Buk $\left(44^{\circ} 12^{\prime} 22^{\prime \prime} \mathrm{N}\right.$, 15043'31"E); Figure 1, nr 25 (Pavlović 2001; Pavlović et al. 2002). Collected in July 1999 by $\mathrm{J}$ Kapelj and G Pavlović in an effort to determine the initial ${ }^{14} \mathrm{C}$ activity and ${ }^{14} \mathrm{C}$ dating of tufa, compared with other tufa deposits (Plitvice Lakes, Krka River). The results are presented in Table 2.

\section{Slovenia}

\section{Krka River Series}

Recent tufa from Krka River, Slovenia (Figure 1, nr 20), was collected and submitted in October 2001 by N Horvatinčić, J Barešić, and A Mihevc, Institute for Karst Investigation, Postojna. Basic water parameters were also measured to study the conditions of tufa precipitation in comparison with tufa in other regions of Dinaric Karst. Results are presented in Table 3.

\section{Podstenjšek Series}

Samples were collected from Podstenjšek brook, a tributary of the Reka, near Ilirska Bistrica, south Slovenia $\left(45^{\circ} 36^{\prime} \mathrm{N}, 14^{\circ} 13^{\prime} \mathrm{E}\right.$; Figure 1, nr 21$)$. Tufa samples from old tufa deposits were also collected (Horvatinčić et al. 2003) in November 1999 by N Horvatinčić and A Mihevc. Results are presented in Table 4.

\section{Bosnia and Herzegovina}

\section{Una River Series}

Tufa from the Una River near Bihać (Figure 1, nr 23), Bosnia and Herzegovina was collected in May 2000 by N Horvatinčić and M Lilić.

\section{Z-2980 Ripački Slap waterfall}

Recent tufa with moss, from waterfalls $\left(44^{\circ} 46^{\prime} \mathrm{N}, 15^{\circ} 57^{\prime} \mathrm{E}\right), 7-8 \mathrm{~km}$ upstream from the town of Bihać. Water: $\mathrm{T}=11.9^{\circ} \mathrm{C} ; \mathrm{pH}=8.2$; conductivity $542 \mu \mathrm{S} / \mathrm{cm}$. 
Table $2{ }^{14} \mathrm{C}$ activities and conventional ${ }^{14} \mathrm{C}$ ages and $\delta^{13} \mathrm{C}$ and $\delta^{18} \mathrm{O}$ values of tufa from Zrmanja River.

\begin{tabular}{llllll}
\hline $\begin{array}{l}\text { Sample } \\
\text { code }\end{array}$ & Sample description & $\mathrm{a}^{14} \mathrm{C}$ & Age & $\delta^{13} \mathrm{C}$ & $\delta^{18} \mathrm{O}$ \\
$(\%)$ & $(\%)$
\end{tabular}

\section{Berberi Buk}

Z-2870 1 - Recent porous wet tufa from barrier, with moss overgrowth, $98.5 \pm 1.5 \quad 125 \pm 120-10.6 \quad-8.0$ in water stream, right bank of Zrmanja River

Z-2871 2 - Recent porous relatively dry tufa from barrier, right bank of $81.1 \pm 1.0 \quad 1680 \pm 100 \quad-9.3 \quad-7.7$ Zrmanja River

\section{Ogari Buk}

Z-2872 1 - Recent porous, very soft tufa, with moss overgrowth. Taken $1.5 \mathrm{~m}$ above water level, $\sim 30-40 \mathrm{~m}$ downstream of the barrier Ogari Buk, right bank of Zrmanja River

Z-2873 2 - Recent porous soft tufa with poor moss overgrowth, $10 \mathrm{~cm}$ above water level. Same location as Z-2872

Z-2874 3 - Dry, porous, soft tufa, from tectonic fissures, 4-5 $\mathrm{m}$ above water level. Same location as Z-2872

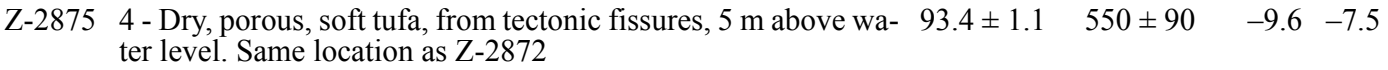

Z-2876 5 - Dry, porous, soft tufa, 10 m above water level. Same location $\quad 98.4 \pm 1.1 \quad 130 \pm 90 \quad-9.6 \quad-7.3$ as Z-2872

Z-2877 6 - Porous, stratified, soft tufa, 10 m above water level. Same lo- $80.8 \pm 1.3 \quad 1710 \pm 130 \quad-10.1 \quad-9.0$ cation as Z-2872

Z-2879 8 - Recent, soft tufa with moss overgrowth, in water stream $\quad 96.7 \pm 1.4 \quad 270 \pm 120-10.6-8.3$ Jankovića Buk

Z-2880 1 - Tufa, surface part hard and compact, lower soft and porous, $\quad 66.3 \pm 1.2 \quad 3300 \pm 140 \quad-10.3 \quad-8.8$ 3-4 $\mathrm{m}$ above water level, $5 \mathrm{~m}$ before Jankovića Buk waterfall, left bank of Zrmanja River

Z-2881 2 - Tufa, inner part compact and hard, outer part soft and porous, $3 \mathrm{~m}$ above water level. Same location as Z-2880

Z-2882 3 - Tufa, soft and porous, partly stratified, $6 \mathrm{~m}$ above water level. Same location as Z-2880

Z-2883 4 - Tufa very porous with moss overgrowth $1 \mathrm{~m}$ above water $\quad 80.2 \pm 1.2 \quad 1770 \pm 120 \quad-10.0 \quad-8.3$ level. Same location as Z-2880

Z-2884 5 - Tufa very porous with shrub facies, 3 m above water level. $\quad 82.3 \pm 1.2 \quad 1560 \pm 120-10.5 \quad-8.6$ Same location as Z-2880

Z-2885 6 - Tufa, surface part hard and compact, below porous and hard, $76.0 \pm 0.7 \quad 2200 \pm 70 \quad-10.2 \quad-7.0$ $6 \mathrm{~m}$ above water level. Same location as Z-2880

Z-2886 7 - Porous, hard tufa, $0.4 \mathrm{~m}$ above water level, from tufa barrier $76.4 \pm 1.1 \quad 2160 \pm 120 \quad-9.8 \quad-7.4$

Table $3{ }^{14} \mathrm{C}$ activities and conventional ${ }^{14} \mathrm{C}$ ages of tufa from Krka River, Slovenia.

\begin{tabular}{|c|c|c|c|}
\hline $\begin{array}{l}\text { Sample } \\
\text { code }\end{array}$ & Sample description & $\begin{array}{l}\mathrm{a}^{14} \mathrm{C} \\
(\mathrm{pMC})\end{array}$ & $\begin{array}{l}\text { Age } \\
\text { (BP) }\end{array}$ \\
\hline Z-3099 & $\begin{array}{l}\text { Krka - Struga - Recent tufa with moss from water stream, } 51 \mathrm{~km} \text { down- } \\
\left.\text { stream from Krka River spring (Struga } 45^{\circ} 50^{\prime} 33^{\prime \prime} \mathrm{N}, 15^{\circ} 14^{\prime} 49^{\prime \prime} \mathrm{E}\right) . \text { Water pa- } \\
\text { rameters: } \mathrm{T}=12.2^{\circ} \mathrm{C} ; \mathrm{pH}=7.8 ; \text { cond: } 441 \mu \mathrm{S} / \mathrm{cm} ; \mathrm{Ca}=3.6 \mathrm{meq} / \mathrm{L} ; \mathrm{Mg}= \\
1.1 \mathrm{meq} / \mathrm{L} \text {; alkalinity } 4.4 \mathrm{meq} / \mathrm{L}\end{array}$ & $76.4 \pm 1.3$ & $2165 \pm 135$ \\
\hline Z-3100 & $\begin{array}{l}\text { Krka - Kot - Dry recent tufa with moss from the wall at the bank river, } 25 \mathrm{~km} \\
\left.\text { downstream from Krka River spring (Dolnji Kot: } 45^{\circ} 47^{\prime} 26^{\prime \prime} \mathrm{N}, 14^{\circ} 58^{\prime} 43^{\prime \prime} \mathrm{E}\right) \text {. } \\
\text { Water parameters: } \mathrm{T}=12.3^{\circ} \mathrm{C} ; \mathrm{pH}=7.6 \text {; cond }=466 \mu \mathrm{S} / \mathrm{cm} ; \mathrm{Ca}=3.5 \mathrm{meq} / \\
\mathrm{L} ; \mathrm{Mg}=1.4 \mathrm{meq} / \mathrm{L} \text {; alkalinity }=4.9 \mathrm{meq} / \mathrm{L}\end{array}$ & $86.3 \pm 1.4$ & $1185 \pm 130$ \\
\hline Z-3101 & $\begin{array}{l}\text { Krka - Dvor 1- Recent tufa with moss from waterfall, } 20 \mathrm{~km} \text { downstream } \\
\text { from Krka River spring }\left(45^{\circ} 48^{\prime} 29^{\prime \prime} \mathrm{N}, 14^{\circ} 57^{\prime} 53^{\prime \prime} \mathrm{E}\right)\end{array}$ & $87.8 \pm 1.2$ & $1045 \pm 115$ \\
\hline Z-3102 & Krka - Dvor 2 - Dry porous tufa from wall of ironworks, 19 th century. & $73.7 \pm 1.1$ & $2455 \pm 120$ \\
\hline Z-3103 & $\begin{array}{l}\text { Krka - Prapreče - Recent tufa from water stream, } 15 \mathrm{~km} \text { downstream from } \\
\text { Krka River spring (Prapreče: } 45^{\circ} 50^{\prime} 08^{\prime \prime} \mathrm{N}, 14^{\circ} 55^{\prime} 09^{\prime \prime} \mathrm{E} \text { ) }\end{array}$ & $88.0 \pm 1.5$ & $1025 \pm 130$ \\
\hline Z-3104 & $\begin{array}{l}\text { Krka - Male Lese }- \text { Recent tufa on a stone in water stream, } 5 \text { km downstream } \\
\text { from Krka River spring }\left(45^{\circ} 52^{\prime} 16^{\prime \prime} \mathrm{N}, 14^{\circ} 48^{\prime} 33^{\prime \prime} \mathrm{E}\right) \text {. Water parameters: } \mathrm{T}= \\
12.7^{\circ} \mathrm{C} ; \mathrm{pH}=7.9 ; \text { cond }=465 \mu \mathrm{S} / \mathrm{cm} ; \mathrm{Ca}=3.4 \mathrm{meq} / \mathrm{L} ; \mathrm{Mg}=1.7 \mathrm{meq} / \mathrm{L} ; \text { al- } \\
\text { kalinity }=4.8 \mathrm{meq} / \mathrm{L}\end{array}$ & $72.4 \pm 1.2$ & $2595 \pm 140$ \\
\hline
\end{tabular}


Table $4{ }^{14} \mathrm{C}$ activities and conventional ${ }^{14} \mathrm{C}$ ages, and $\delta^{13} \mathrm{C}$ and $\delta^{18} \mathrm{O}$ values of tufa from Podstenjšek brook, Slovenia.

\begin{tabular}{|c|c|c|c|c|c|}
\hline $\begin{array}{l}\text { Sample } \\
\text { code }\end{array}$ & Sample description & $\begin{array}{l}\mathrm{a}^{14} \mathrm{C} \\
(\mathrm{pMC})\end{array}$ & $\begin{array}{l}\text { Age } \\
(\mathrm{BP})\end{array}$ & $\begin{array}{l}\delta^{13} \mathrm{C} \\
(\%)\end{array}$ & $\begin{array}{l}\delta^{18} \mathrm{O} \\
(\%)\end{array}$ \\
\hline Z-2914 & enjšek 1 - Dry tufa & $32.3 \pm 0.7$ & $9090 \pm$ & -10.7 & -7.0 \\
\hline Z-2915 & barrier, second terrace, $0.5 \mathrm{~m}$ below sur- & $45.1 \pm 0.9$ & $6395 \pm 160$ & -11.3 & -6.6 \\
\hline Z-2916 & $\begin{array}{l}\text { Podstenjšek } 3 \text { - Tufa barrier, second terrace, } 1.5 \mathrm{~m} \text { below sur- } \\
\text { face, near the bridge }\end{array}$ & $44.2 \pm 0.7$ & $6550 \pm 120$ & -11.3 & -6.4 \\
\hline Z-2917 & $\begin{array}{l}\text { jšek } 4 \text { - Tufa barrier, third terrace, } 0.5 \mathrm{~m} \text { below the } \\
\text { near the brook }\end{array}$ & $53.9 \pm 1.0$ & $4970 \pm 145$ & -11.2 & -6.2 \\
\hline Z-2918 & & & 686 & -11.4 & -6.4 \\
\hline Z-2919 & Podstenjšek 6 - Recent tufa in water stream, near third barrier & $97.5 \pm 1.0$ & $200 \pm 80$ & -11.8 & -6.8 \\
\hline
\end{tabular}

\section{Z-2981 Četića Mill 1}

$2910 \pm 110$ $69.6 \pm 0.9 \mathrm{pMC}$

Dry tufa, partly soft and porous, on the river bank at Četića Mill, in Bihać. Water: $\mathrm{T}=12.5^{\circ} \mathrm{C} ; \mathrm{pH}=$ 8.2; conductivity $535 \mu \mathrm{S} / \mathrm{cm}$.

\section{Z-2982 Četića Mill 2}

$\delta^{13} \mathrm{C}=-9.4 \%$

Soft, partly porous tufa, $0.5 \mathrm{~m}$ below Z-2981.

\section{Z-2983 Četića Mill 3}

Recent tufa with moss from water stream. Same location as Z-2981.

\section{Z-2984 Kostena 1}

Old tufa barrier, $6 \mathrm{~m}$ high, $20 \mathrm{~m}$ from Una River. Hard, partly porous tufa, $2 \mathrm{~m}$ from the bottom of barrier $17 \mathrm{~km}$ downstream from Bihać.

\section{Z-2985 Kostena 2}

Tufa 1 m below Z-2984.

Turkey

\section{Denizli Tufa Series}

Tufa from 3 sites and associated spring waters were investigated in Denizli Province, west Turkey: Honaz, Gunay, and Sakizcilar (Table 5). The aim was to compare the conditions of tufa formation from different hydrogeological sites using physico-chemical parameters of water and isotopic composition of tufa, and to compare these results with those of tufa deposits in Dinaric Karst. Samples were collected by M Özkul and A Gökgöz, Pamukkale University, Denizli, and N Horvatinčić in October 2003 (Horvatinčić et al. 2005; Özkul et al. 2010). 
Table $5{ }^{14} \mathrm{C}$ activities and conventional ages and $\delta^{13} \mathrm{C}$ and $\delta^{18} \mathrm{O}$ values of tufa from Denizli Province, Turkey.

\begin{tabular}{|c|c|c|c|c|c|}
\hline $\begin{array}{l}\text { Sample } \\
\text { code }\end{array}$ & Sample description & $\begin{array}{l}\mathrm{a}^{14} \mathrm{C} \\
(\mathrm{pMC})\end{array}$ & $\begin{array}{l}\text { Age } \\
(\mathrm{BP})\end{array}$ & $\begin{array}{l}\delta^{13} \mathrm{C} \\
(\%)\end{array}$ & $\begin{array}{l}\delta^{18} \mathrm{O} \\
(\%)\end{array}$ \\
\hline & Honaz & & & & \\
\hline Z-3414 & $\begin{array}{l}\text { Colassea } 1 \text { - Huge tufa deposit, dry, not more active; hard, } \\
\text { porous, partly covered by grass }\end{array}$ & $15.1 \pm 0.3$ & $15,200 \pm 135$ & 2.9 & -8.1 \\
\hline Z-3415 & $\begin{array}{l}\text { Colassea } 2 \text { - Old tufa deposit, near the brook, height } \sim 10 \mathrm{~m} \text {, } \\
\text { porous, hard tufa }\end{array}$ & $16.9 \pm 0.2$ & $14,300 \pm 85$ & 3.4 & -7.7 \\
\hline Z-3416 & Degirmenler 1 - Recent tufa from water, soft & $20.0 \pm 1.2$ & $12,940 \pm 485$ & -0.9 & -9.4 \\
\hline Z-3417 & $\begin{array}{l}\text { Degirmenler } 2 \text { - Recent tufa with moss from waterfall, soft, } \\
\text { similar as Z-3416 }\end{array}$ & $21.1 \pm 0.3$ & $12,505 \pm 105$ & -0.4 & -9.2 \\
\hline Z-3418 & $\begin{array}{l}\text { Kayalti } 1 \text { - Tufa barrier, } 20 \mathrm{~m} \text { high, not more active; soft, } \\
\text { porous; taken } 1 \mathrm{~m} \text { above the bottom }\end{array}$ & $7.5 \pm 0.1$ & $20,850 \pm 140$ & 1.8 & -10.0 \\
\hline Z-3419 & $\begin{array}{l}\text { Kayalti } 2 \text { - Same location as Z-3418; taken } \sim 10 \mathrm{~m} \text { above Z- } \\
3418 \text {, from the "cave" }\end{array}$ & $38.1 \pm 0.3$ & $7750 \pm 60$ & 0.1 & -8.2 \\
\hline Z-3420 & Kayalti 3 - Same location as Z-3419, different form & $23.5 \pm 0.2$ & $11,630 \pm 70$ & 1.0 & -8.5 \\
\hline Z-3421 & $\begin{array}{l}\text { Guney } \\
\text { Waterfall } 1 \text { - Recent tufa with moss, above 1. big waterfall } \\
\text { (recent precipitation) }\end{array}$ & $63.8 \pm 0.5$ & $3610 \pm 60$ & -9.1 & -8.4 \\
\hline Z-3422 & $\begin{array}{l}\text { Waterfall } 2 \text { - Left side of the waterfall; looks like old tufa, } \\
\text { porous, relatively soft }\end{array}$ & $64.1 \pm 0.3$ & $3565 \pm 70$ & -7.7 & -8.2 \\
\hline Z-3423 & $\begin{array}{l}\text { Waterfall } 3 \text { - Recent tufa with moss, below } 1 \text {. big waterfall } \\
\text { (from the water) }\end{array}$ & $65.7 \pm 0.5$ & $3375 \pm 60$ & -7.7 & -7.9 \\
\hline Z-3424 & $\begin{array}{l}\text { Waterfall } 4 \text { - Recent tufa with moss, } \sim 10 \text { m below Z-3423, } \\
\text { same waterfall }\end{array}$ & $65.5 \pm 0.5$ & $3395 \pm 60$ & -7.8 & -8.1 \\
\hline Z-3425 & Waterfall 5 - Left side of the big waterfall, dry, compact & $76.0 \pm 0.4$ & $2200 \pm 45$ & -6.2 & -8.1 \\
\hline Z-3426 & $\begin{array}{l}\text { Waterfall } 6 \text { - Dry tufa, on the hill above big waterfall, rela- } \\
\text { tively soft, porous }\end{array}$ & $73.5 \pm 0.6$ & $2465 \pm 70$ & -6.0 & -7.4 \\
\hline Z-3427 & Waterfall 7 - Fragment of tufa, dry, close to Z-3426 & $68.0 \pm 2.1$ & $3095 \pm 240$ & -8.0 & -7.6 \\
\hline Z-3428 & Waterfall 8 - Dry tufa barrier, above Z-3427 & $62.7 \pm 1.9$ & $3750 \pm 250$ & -8.4 & -8.1 \\
\hline Z-3429 & $\begin{array}{l}\text { Waterfall } 9 \text { - Dry tufa barrier above Z-3428, highest level at } \\
\text { this area; hard, compact tufa }\end{array}$ & $48.6 \pm 0.5$ & $5790 \pm 80$ & -7.7 & -8.1 \\
\hline Z-3430 & $\begin{array}{l}\text { Waterfall } 10 \text { - Dry tufa barrier near road, below other tufa } \\
\text { level (from above) }\end{array}$ & $65.0 \pm 0.6$ & $3460 \pm 70$ & -8.2 & -8.0 \\
\hline Z-3431 & Waterfall 11 - Dry tufa barrier near road, below Z-3430 & $67.9 \pm 1.8$ & $3110 \pm 215$ & -6.5 & -7.6 \\
\hline \multirow[t]{2}{*}{ Z-3432 } & $\begin{array}{l}\text { Waterfall } 12 \text { - Recent tufa with moss, from the lowest wa- } \\
\text { terfall }\end{array}$ & $70.6 \pm 0.6$ & $2800 \pm 70$ & -6.9 & -7.9 \\
\hline & Sakizcilar Village & & & & \\
\hline Z-3433 & Sakizcilar Village 1 - Recent tufa below moss, waterfall & $77.3 \pm 2.0$ & $2060 \pm 210$ & -9.6 & -8.2 \\
\hline Z-3434 & Sakizcilar Village 2 - Dry tufa from left side of waterfall & $90.1 \pm 2.2$ & $830 \pm 190$ & -5.7 & -7.1 \\
\hline
\end{tabular}

China

\section{Guangxi Zhuang Series}

Tufa from the karst region of Guangxi Zhuang Autonomous Region, south China $\left(24^{\circ} \mathrm{N}, 108^{\circ} \mathrm{E}\right)$. Collected in 1999 by Y Daoxian, Institute of Karst Geology, Guilin, and submitted by H Bilinski, Rudjer Bošković Institute (Frančišković-Bilinski et al. 2003).

Z-3007 Fengshan Nz-1 $\delta^{13} \mathrm{C}=-6.5 \%, \delta^{18} \mathrm{O}=-9.1 \%$ o

Tufa Nz-1, karst spring from a fissure, medium level, Fengshan County.

Z-3008 Fengshan Nz-2 $\delta^{13} \mathrm{C}=-9.3 \%$ o $\delta^{18} \mathrm{O}=-\mathbf{9 . 5 \%}$

Tufa Nz-2, karst spring from a fissure, lower level, Fengshan County.
$980 \pm 70$ $88.3 \pm 0.7 \mathrm{pMC}$

$108.2 \pm 1.2 \mathrm{pMC}$ 
Z-3009 Mashan No. 2

Tufa, small karst spring, lower level, Mashan County.

Z-3011 Mashan No. 3

$8290 \pm 160$

$\delta^{13} \mathrm{C}=-11.9 \%, \delta^{18} \mathrm{O}=-8.9 \%$

Tufa, small karst spring, medium level, Mashan County.

$1330 \pm 100$

Z-3010 Linyun No. 3

$84.7 \pm 1.0$ pMC

Tufa, natural karst well, Linyun County.

\section{Soil and Sediment Samples}

Z-2749 Mljet

$4055 \pm 110$

$60.0 \pm 0.8 \mathrm{pMC}$

Black layer of wet soil from a 103-cm-long core, layer 25-30 cm, channel Soline, from a connection between the open sea and Mljet Lakes on Mljet Island, Adriatic Sea, northwest from Dubrovnik, Dalmatia, Croatia (42 $\left.46^{\prime} 01^{\prime \prime} \mathrm{N}, 1^{\circ} 22^{\prime} 50^{\prime \prime} \mathrm{E}\right)$ (Govorčin et al. 2001). Collected in October 1997 by M Juračić, Faculty of Natural Sciences, Zagreb; cal age 2864-2460 cal BC.

\section{Z-2363 Bol}

$30,100 \pm 2000$

$2.3 \pm 0.6 \mathrm{pMC}$

Clayey sand at Zlatni Rat, Bol on Brač Island, Adriatic Sea, Croatia (43 $\left.15^{\prime} 24^{\prime \prime} \mathrm{N}, 16^{\circ} 38^{\prime} 05^{\prime \prime} \mathrm{E}\right)$, submitted in 1990 by Lj Marjanac.

\section{Postojna Soil Series}

Soil depth profiles from 2 locations: Nemčji Vrh $\left(45^{\circ} 48^{\prime} \mathrm{N}, 14^{\circ} 12^{\prime} \mathrm{E}\right)$ and Direkcija $\left(45^{\circ} 47^{\prime} \mathrm{N}\right.$, $14^{\circ} 12^{\prime} \mathrm{E}$ ) above the Postojna Cave, Slovenia (Figure 1, nr 11). Collected in October 1996 by B Vokal (Vokal 1999). Results are presented in Table 6.

Table $6{ }^{14} \mathrm{C}$ activities and $\delta^{13} \mathrm{C}$ values of soil samples from locations Nemčji Vrh and Direkcija.

\begin{tabular}{llcll}
\hline $\begin{array}{l}\text { Sample } \\
\text { code }\end{array}$ & Location & $\begin{array}{l}\text { Soil depth } \\
(\mathrm{cm})\end{array}$ & $\begin{array}{l}\mathrm{a}^{14} \mathrm{C} \\
(\mathrm{pMC})\end{array}$ & $\begin{array}{l}\delta^{13} \mathrm{C} \\
(\%)\end{array}$ \\
\hline Z-2689 & Nemčji Vrh & $0-2$ & $119.1 \pm 1.2$ & -26.6 \\
Z-2690 & Nemčji Vrh & $3-5$ & $117.7 \pm 1.7$ & -27.1 \\
Z-2691 & Nemčji Vrh & $5-10$ & $108.8 \pm 1.1$ & -27.4 \\
Z-2683 & Direkcija & $0-2$ & $117.1 \pm 1.7$ & -27.0 \\
Z-2684 & Direkcija & $2-7$ & $110.5 \pm 1.6$ & -27.3 \\
Z-2688 & Direkcija & $22-30$ & $97.6 \pm 1.0$ & -26.4 \\
\hline
\end{tabular}

\section{Lake Kozjak Sediment - Organic Fraction Series}

Lake sediment was collected from Kozjak Lake, National Park Plitvice Lakes, at $21.5 \mathrm{~m}$ water depth (Figure 1, nr 22). An organic fraction of lake sediment was obtained after acid dissolution of carbonate fraction, which was previously dated (Horvatinčić et al 1999) and the results are given as comments. Samples were collected in August 1990 by D Srdoč and N Horvatinčić.

Z-2319 Lake Kozjak 1, 1-5 cm depth

$111.3 \pm 1.6 \mathrm{pMC}$ $\delta^{13} \mathrm{C}=-33.6 \%$

Carbonate fraction: $88.6 \pm 0.8$ pMC (Z-2233). 
Z-2317 Lake Kozjak 2, 5-10 cm depth

$660 \pm 120$

$\delta^{13} \mathrm{C}=-29.9 \%$

$91.6 \pm 1.4 \mathrm{pMC}$

Carbonate fraction: $74.7 \pm 0.8 \mathrm{pMC}(\mathrm{Z}-2234)$.

Z-2316 Lake Kozjak 3, 10-15 cm depth

$108.4 \pm 1.4 \mathrm{pMC}$

$\delta^{13} \mathrm{C}=-30.2 \%$

Carbonate fraction: $72.6 \pm 0.8 \mathrm{pMC}$ (Z-2235).

Z-2318 Lake Kozjak 4, 15-20 cm depth

$\delta^{13} \mathrm{C}=-30.2 \%$

$114.0 \pm 1.5 \mathrm{pMC}$

Carbonate fraction: $71.4 \pm 0.5 \mathrm{pMC}$ (Z-2236).

BIOLOGICAL SAMPLES

Mollusks/Shells

Z-2368 Mljet, S-2(o)

$4770 \pm 170$

$54.9 \pm 1.1 \mathrm{pMC}$

Marine mollusks, Mljet Island, northwest from Dubrovnik, south Dalmatia, Croatia $\left(42^{\circ} 45^{\prime} \mathrm{N}\right.$, $17^{\circ} 44^{\prime} 30^{\prime \prime} \mathrm{E}$ ), were collected in 1991 by V Klein.

\section{Rava Mollusks series}

Various mollusks from Lokvina Bay, Rava Island, Zadar Archipelago, Dalmatia, Croatia $\left(44^{\circ} 02^{\prime} 21^{\prime \prime} \mathrm{N}, 15^{\circ} 03^{\prime} 29^{\prime \prime} \mathrm{E}\right)$ were collected and submitted in February 2001 by M Surić for a comparison with biogenic overgrowth (cf. series Brač, Pag, and Rogoznica).

\section{Z-3065 Rava, SH-1}

$107.5 \pm 1.2 \mathrm{pMC}$

$\delta^{13} \mathrm{C}=-1.0 \%$, $\delta^{18} \mathrm{O}=-0.1 \%$ o

Murex (Murex brandaris).

Z-3071 Rava, SH-4

$106.9 \pm 1.7 \mathrm{pMC}$

$\delta^{13} \mathrm{C}=+0.6 \%, \delta^{18} \mathrm{O}=+0.3 \%$ o

Oysters (Crassostrea gigas).

\section{Z-2435 Rogoznica}

Mollusks from sediment in Rogoznica, near Šibenik, Dalmatia, Croatia $\left(43^{\circ} 31^{\prime} 42^{\prime \prime} \mathrm{N}, 15^{\circ} 57^{\prime} 58^{\prime \prime} \mathrm{E}\right)$, were collected in November 1992 by D Petricioli.

\section{Z-3120 Mussels from Zadar 1}

$100.3 \pm 1.1 \mathrm{pMC}$ Mussels (Mytilus edulis) from the Adriatic Sea, near Zadar, Croatia, were collected in 2002 by M Surić and measured by GPC.

Z-3300 Mussels from Zadar 2

$100.4 \pm 0.4 \mathrm{pMC}$

Mussels (Mytilus edulis); same as Z-3120; measured by LSC. Sample used as secondary standard for direct absorption technique (Horvatinčić et al. 2004)

\section{Botanical Samples}

\section{Medvednica Series}

Leaves were collected by N Horvatinčić at the top of Medvednica Mountain (1035 m asl), location Puntijarka, north of Zagreb, Croatia $\left(45^{\circ} 54^{\prime} 14^{\prime \prime} \mathrm{N}, 15^{\circ} 58^{\prime} 09^{\prime \prime} \mathrm{E}\right)$ for a comparison with the atmospheric $\mathrm{CO}_{2}{ }^{14} \mathrm{C}$ activity. 
Z-2599 Medvednica, Leaves 1

$111.9 \pm 1.2 \mathrm{pMC}$

Leaves collected in December 1995. Atmospheric $\mathrm{CO}_{2}$ October-November 1995: 112.9 $\pm 1.4 \mathrm{pMC}$ at the same location.

Z-2679 Medvednica, Leaves 2

$110.9 \pm 1.7 \mathrm{pMC}$

Leaves collected in November 1996. Atmospheric $\mathrm{CO}_{2}$ September-October 1996: 110.7 $\pm 1.5 \mathrm{pMC}$ at the same location.

Z-2606 Zaprešić, October 1995

$110.0 \pm 1.1 \mathrm{pMC}$

Flowers from a balcony in the town of Zaprešić near Zagreb, Croatia $\left(45^{\circ} 51^{\prime} \mathrm{N}, 15^{\circ} 48^{\prime} \mathrm{E}\right)$ were collected in October 1995 by I Krajcar Bronić. Comparison with mean annual activity of atmospheric $\mathrm{CO}_{2}$ : 111.1 pMC for Northern Hemisphere (Levin and Kromer 2004); $111.8 \mathrm{pMC}$ in Zagreb; 112.3 pMC at Medvednica Mountain (Krajcar Bronić et al. 1998, 2010).

\section{Postojna Series}

Leaves near Postojna Cave, Slovenia $\left(45^{\circ} 47^{\prime} \mathrm{N}, 1^{\circ} 12^{\prime} \mathrm{E}\right)$ were collected in October 1996 by B Vokal, Jožef Stefan Institute, Ljubljana, Slovenia, within a comprehensive study of isotopic composition of various environmental samples in and around Postojna Cave (Vokal 1999).

Z-2680 Postojna, Leaves 1

$116.3 \pm 1.2 \mathrm{pMC}$

In front of Old Direction of Postojna Cave.

Z-2681 Postojna, Leaves 2

$111.8 \pm 1.7 \mathrm{pMC}$

In front of New Direction of Postojna Cave.

Z-2682 Postojna, Leaves 3

$113.0 \pm 1.7 \mathrm{pMC}$

Nemčji Vrh.

Z-2261 Vinča

$114.3 \pm 1.8 \mathrm{pMC}$

Different annual plants from Vinča near Belgrade, Serbia $\left(44^{\circ} 50^{\prime} \mathrm{N}, 20^{\circ} 20^{\prime} \mathrm{E}\right)$ were submitted in May 1990 by M Hadžišehović, Nuclear Institute Vinča, Belgrade, during a comparison with mean annual

${ }^{14} \mathrm{C}$ activity of atmospheric $\mathrm{CO}_{2}: 114.8 \mathrm{pMC}$ for Northern Hemisphere (Levin and Kromer 2004).

\section{HYDROGEOLOGICAL SAMPLES}

Croatia

Z-2986 Bistra - Budinščina

$3180 \pm 105$

$67.3 \pm 0.9 \mathrm{pMC}$

Spring water Bistra, Gotalovec near Budinščina $\left(46^{\circ} 07^{\prime} 43^{\prime \prime} \mathrm{N}, 16^{\circ} 12^{\prime} 20^{\prime \prime} \mathrm{E}\right)$, NW Croatia, was submitted in July 2000 by J Vrbanek, Gotalka Ltd., Budinščina. Comment (JV): Survey before expoitation.

\section{Jamnica Series}

Groundwater from Jamnica near Pokupsko $\left(45^{\circ} 33^{\prime} \mathrm{N}, 15^{\circ} 51^{\prime} \mathrm{E}\right)$, central Croatia, was collected in July 1999 and submitted by B Briški. Comment (BB): Survey before exploitation of bottled mineral water. Results are presented in Table 7.

\section{Lipik Series}

Water samples from Lipik, W Slavonia, Croatia $\left(45^{\circ} 25^{\prime} \mathrm{N}, 17^{\circ} 10^{\prime} \mathrm{E}\right)$, were submitted in January 1999 by I Mami, Food Company Podravka, Koprivnica. 
Table $7{ }^{14} \mathrm{C}$ activities and conventional ages, bicarbonate concentration and tritium activities (A) of groundwaters from Jamnica.

\begin{tabular}{lllllll}
\hline Lab nr & Sample code & $\begin{array}{l}\mathrm{HCO}_{3} \text { conc. } \\
(\mathrm{mg} / \mathrm{L})\end{array}$ & $\begin{array}{l}\mathrm{a}^{14} \mathrm{C} \\
(\mathrm{pMC})\end{array}$ & $\begin{array}{l}\text { Age } \\
(\mathrm{BP})\end{array}$ & $\begin{array}{l}\text { Tritium } \\
\text { lab nr }\end{array}$ & $\begin{array}{l}\mathrm{A} \\
(\mathrm{Bq} / \mathrm{L})\end{array}$ \\
\hline Z-2861 & Sample 13 & 384 & $67.8 \pm 1.2$ & $3120 \pm 145$ & $\mathrm{~T}-2404$ & $1.02 \pm 0.12$ \\
Z-2862 & Sample 14a & 378 & $80.9 \pm 1.3$ & $1700 \pm 130$ & $\mathrm{~T}-2405$ & $1.50 \pm 0.12$ \\
Z-2863 & Sample 18 & 984 & $1.5 \pm 0.8$ & $33,520 \pm 4550$ & $\mathrm{~T}-2406$ & $<0.11$ \\
Z-2864 & Sample 19 & 927 & $19.0 \pm 0.6$ & $13,325 \pm 260$ & $\mathrm{~T}-2407$ & $0.60 \pm 0.13$ \\
Z-2865 & Sample 24 & 979 & $57.9 \pm 1.1$ & $4385 \pm 150$ & $\mathrm{~T}-2408$ & $1.53 \pm 0.18$ \\
Z-2866 & Sample 2 & 579 & $43.4 \pm 0.7$ & $6705 \pm 130$ & $\mathrm{~T}-2409$ & $1.15 \pm 0.20$ \\
Z-2867 & Sample 8 & 5230 & $<0.7$ & & $\mathrm{~T}-2410$ & $<0.11$ \\
\hline
\end{tabular}

\section{Z-2839 Well B-7}

Mineral water; tritium activity $<0.1 \mathrm{~Bq} / \mathrm{L}(\mathrm{T}-2383)$.

\section{Z-2840 Well Kukunje}

Groundwater; tritium activity $<0.1 \mathrm{~Bq} / \mathrm{L}(\mathrm{T}-2384)$.

\section{Zagreb Geothermal Waters Series}

Geothermal water samples from aquifers near Zagreb, NW Croatia, were submitted in October 1997 by S Kapelj and M Kovačić, Institute of Geology, during a hydrogeochemical and isotopic study of geothermal aquifers due to exploitation of geothermal energy in Zagreb area (Kovačić et al. 1998). Results are presented in Table 8.

Table $8{ }^{14} \mathrm{C}$ activities and conventional ages of thermal waters from various spas near Zagreb, Croatia.

\begin{tabular}{lllr}
\hline Lab nr & Sample name & $\begin{array}{l}\mathrm{a}^{14} \mathrm{C} \\
(\mathrm{pMC})\end{array}$ & \multicolumn{1}{c}{$\begin{array}{l}\text { Age } \\
(\mathrm{BP})\end{array}$} \\
\hline Z-2744 & Sutinska Vrela, SV/97, Podsused $\left(45^{\circ} 49^{\prime} \mathrm{N}, 15^{\circ} 50^{\prime} \mathrm{E}\right)$ & $74.4 \pm 1.3$ & $2325 \pm 140$ \\
$\mathrm{Z}-2745$ & Topličica, Gornja Dubravica, TD/97 $\left(45^{\circ} 57^{\prime} \mathrm{N}, 15^{\circ} 44^{\prime} \mathrm{E}\right)$ & $89.1 \pm 1.6$ & $875 \pm 150$ \\
$\mathrm{Z}-2746$ & Sports center “Mladost,” M/97, Hole PDT2, Zagreb $\left(45^{\circ} 49^{\prime} \mathrm{N}\right.$, & $11.0 \pm 0.8$ & $11,650 \pm 620$ \\
& $\left.15^{\circ} 50^{\prime} \mathrm{E}\right)$ & $1.4 \pm 0.8$ & $34,300 \pm 3500$ \\
\hline
\end{tabular}

\section{Zagreb Aquifers Series}

Water samples from the aquifers Kosnica, Črnkovec, Jakuševec, and Vrbovec near Zagreb, in the Sava River basin, were collected in September 2001 and February 2002 by S Kapelj during a survey of groundwater and Sava River water for possible exploitation for water supply. Results are presented in Table 9.

\section{Slovenia}

\section{Z-2438 Hrastnik}

Water from the cave Kotredež, chamber A, borehole $4 \mathrm{a}$, Hrastnik, central Slovenia $\left(46^{\circ} 09^{\prime} \mathrm{N}\right.$, $15^{\circ} 05^{\prime} \mathrm{E}$ ), was collected in January 1993 by J Prestor, Geological Survey of Slovenia, Ljubljana. Tritium activity: $0.68 \pm 0.12 \mathrm{~Bq} / \mathrm{L}$ (T-1983). 
Table $9{ }^{14} \mathrm{C}$ activities and tritium activities (A) of groundwaters from aquifers Kosnica, Črnkovec, Jakuševec and Vrbovec near Zagreb, Croatia.

\begin{tabular}{llrll}
\hline Lab nr & Sample name & $\begin{array}{l}\mathrm{a}^{14} \mathrm{C} \\
(\mathrm{pMC})\end{array}$ & $\begin{array}{l}\text { Tritium } \\
\text { lab nr }\end{array}$ & $\begin{array}{l}\mathrm{A} \\
(\mathrm{Bq} / \mathrm{L})\end{array}$ \\
\hline & September 2001 & & & \\
Z-3085 & Kosnica ČDP-9/1-IX-01 & $102.0 \pm 0.9$ & $\mathrm{~T}-2610$ & $2.03 \pm 0.27$ \\
Z-3086 & Kosnica ČDP-9/2-IX-01 & $95.4 \pm 1.1$ & $\mathrm{~T}-2611$ & $1.98 \pm 0.28$ \\
Z-3087 & Ǩsnica ČDP-9/3-IX-01 & $87.1 \pm 1.0$ & $\mathrm{~T}-2612$ & $1.61 \pm 0.28$ \\
Z-3088 & Črnkovec ČDP-23/1-IX/01 & $84.1 \pm 1.4$ & $\mathrm{~T}-2613$ & $2.67 \pm 0.30$ \\
Z-3089 & Črnkovec ČDP-23/2-IX/01 & $86.4 \pm 1.0$ & $\mathrm{~T}-2614$ & $2.48 \pm 0.29$ \\
Z-3090 & Črnkovec ČDP-23/3-IX/01 & $94.7 \pm 1.5$ & $\mathrm{~T}-2615$ & $2.00 \pm 0.29$ \\
Z-3091 & Jakuševec JP-10-IX/01 & $97.6 \pm 1.5$ & $\mathrm{~T}-2616$ & $4.06 \pm 0.32$ \\
Z-3092 & Vrbovec NOS-28-IX/01 & $2.5 \pm 0.6$ & $\mathrm{~T}-2617$ & $0.33 \pm 0.15$ \\
Z-3093 & Vrbovec NOS-28A-IX/01 & $91.9 \pm 1.5$ & $\mathrm{~T}-2618$ & $1.75 \pm 0.29$ \\
Z-3094 & Sava River-IX/01 & $89.6 \pm 1.4$ & $\mathrm{~T}-2619$ & $1.21 \pm 0.28$ \\
& February 2002 & & & \\
Z-3121 & Kosnica ČDP-9/1-2/02 & $101.5 \pm 1.4$ & $\mathrm{~T}-2721$ & $1.79 \pm 0.24$ \\
Z-3122 & Kosnica ČDP-9/2-2/02, & $100.8 \pm 1.5$ & $\mathrm{~T}-2722$ & $0.94 \pm 0.24$ \\
Z-3123 & Kosnica ČDP-9/3-2/02 & - & $\mathrm{T}-2723$ & $0.73 \pm 0.23$ \\
Z-3124 & Črnkovec ČDP-23/1-2/02 & $82.5 \pm 1.2$ & $\mathrm{~T}-2724$ & $2.36 \pm 0.25$ \\
Z-3125 & Črnkovec ČDP-23/2-2/02 & $92.2 \pm 1.4$ & $\mathrm{~T}-2725$ & $1.84 \pm 0.24$ \\
Z-3126 & Črnkovec ČDP-23/3-2/02 & $94.4 \pm 1.3$ & $\mathrm{~T}-2726$ & $1.54 \pm 0.24$ \\
Z-3127 & Jakuševec JP-10-2/02 & $98.3 \pm 1.3$ & $\mathrm{~T}-2727$ & $3.32 \pm 0.25$ \\
Z-3128 & Vrbovec NOS-28-2/02 & $1.6 \pm 0.5$ & $\mathrm{~T}-2728$ & $<0.23$ \\
Z-3129 & Vrbovec NOS-28A-XI/01 & $88.6 \pm 1.2$ & $\mathrm{~T}-2729$ & $1.29 \pm 0.24$ \\
Z-3130 & Sava River-X/01 & $99.5 \pm 1.4$ & $\mathrm{~T}-2730$ & $0.45 \pm 0.23$ \\
\hline
\end{tabular}

\section{Mežica Water Series}

Groundwater from Mežica, N Slovenia $\left(46^{\circ} 31^{\prime} \mathrm{N}, 14^{\circ} 51^{\prime} \mathrm{E}\right)$, was collected in February 2003 by $\mathrm{J}$ Prestor to study the groundwater dynamics in aquifers in the area of Meža River.

Z-2442 Mošenik-1

Borehole water in Triassic limestone. $\mathrm{pH}: 7.7 ; \mathrm{HCO}_{3}^{--}: 340 \mathrm{mg} / \mathrm{L}$; tritium activity $1.85 \pm 0.16 \mathrm{~Bq} / \mathrm{L}$ (T-1989).

\section{Z-2443 NAVR-1}

$3450 \pm 160$

$65.0 \pm 1.3 \mathrm{pMC}$

Groundwater from spring in Triassic limestone. $\mathrm{pH}: 7.6 ; \mathrm{HCO}_{3}{ }^{-}: 220 \mathrm{mg} / \mathrm{L}$; tritium activity $2.44 \pm$ $0.16 \mathrm{~Bq} / \mathrm{L}(\mathrm{T}-1990)$.

\section{Z-2444 UN-1}

Groundwater from spring in Triassic limestone/dolomites. $\mathrm{pH}: 7.7 ; \mathrm{HCO}_{3}{ }^{-}: 250 \mathrm{mg} / \mathrm{L}$; tritium activity $2.12 \pm 0.22 \mathrm{~Bq} / \mathrm{L}(\mathrm{T}-1991)$.

\section{Z-2445 Graben 1}

Groundwater from spring in dolomite limestone. $\mathrm{pH}: 7.6 ; \mathrm{HCO}_{3}^{-}: 230 \mathrm{mg} / \mathrm{L}$; tritium activity: $2.95 \pm$ $0.16 \mathrm{~Bq} / \mathrm{L}(\mathrm{T}-1992)$. 


\section{ACKNOWLEDGMENTS}

We thank M A Geyh and A Suckow (Leibniz Institute for Applied Geosciences, Hannover, Germany) for stable isotope measurements; and E Hernaus, B Mustač, and A Rajtarić for sample preparation. Work prepared under the Project Grant 098-0982709-2741 from the Ministry of Science, Education and Sports of the Republic of Croatia.

\section{REFERENCES}

Bronk Ramsey C. 2009. Bayesian analysis of radiocarbon dates. Radiocarbon 51(1):337-60.

Bronk Ramsey Ch. 2012. OxCal v. 4.1, The Oxford Radiocarbon Accelerator Unit, University of Oxford. URL: https://c14.arch.ox.ac.uk/oxcal. Accessed 26 January 2012.

Frančišković-Bilinski S, Bilinski H, Barišić D, Horvatinčić N, Daoxian Y. 2003. Analysis of karst tufa from Guangxi Province (China). Acta Geologica Sinica 77(2):267-75.

Govorčin D, Juračić M, Horvatinčić N, Onofri V. 2001. Holocene sedimentation in the Soline Channel (Mljet Lakes, Adriatic Sea). Natura Croatica 10(4):247-58.

Horvatinčić N, Božić V. 2001. Ledena jama na Velebitu izazov znanstvenicima. Speleolog - $\breve{C}$ asopis za speleologiju 46/47:47-52. In Croatian, extended abstract in English.

Horvatinčić N, Srdoč D, Obelić B, Krajcar Bronić I. 1990. Radiocarbon dating of intercomparison samples at the Zagreb Radiocarbon Laboratory. Radiocarbon 32(3):295-300.

Horvatinčić N, Obelić B, Krajcar Bronić I, Srdoč D, Čalić R. 1999. Rudjer Bošković Institute radiocarbon measurements XIV. Radiocarbon 41(2):199-213.

Horvatinčić N, Krajcar Bronić I, Obelić B. 2003. Differences in the ${ }^{14} \mathrm{C}$ age, $\delta^{13} \mathrm{C}$ and $\delta^{18} \mathrm{O}$ of Holocene tufa and speleothems in the Dinaric Karst. Palaeogeography, Palaeoclimatology, Palaeoecology 193(1):13957.

Horvatinčić N, Barešić J, Krajcar Bronić I, Obelić B. 2004. Measurement of low ${ }^{14} \mathrm{C}$ activities in a liquid scintillation counter in the Zagreb Radiocarbon Laboratory. Radiocarbon 46(1):105-16.

Horvatinčić N, Barešić J, Özkul M, Gökgöz A. 2005. Isotopic and geochemical investigation of tufa in Denizli province, Turkey. In: Özkul M, Yagiz S, Jones B, editors. Proceedings of 1st International Symposium on Travertine, Denizli, Turkey. Denizli: Kozan Ofset Matbaacılık San. ve Tic. Ltd. Ankara. p 162-70.

Jelinić I, Horvatinčić N, Božić V. 2001. Ledena jama u Lomskoj dulibi. Senjski zbornik 28:5-20. In Croatian with English abstract.

Kapelj J. 2002. Strukturni sklop šireg područja Promine u sjevernoj Dalmaciji i odraz na hidrogeološke odnose [Structural framework of the northern Dalmatia and its effect on hydrogeological conditions]. PhD thesis. Zagreb: Faculty of Natural Sciences. 137 p.

Kovačić M, Kapelj S, Perica R, Horvatinčić N. 1998. Exploration of geothermal waters in the area of Zagreb by hydrogeochemical methods. In: Petraš J, editor. Proceedings of International Symposium on Water Management and Hydraulic Engineering, Dubrovnik. Zagreb.

Krajcar Bronić I, Horvatinčić N, Srdoč D, Obelić B. 1992. Experimental determination of the ${ }^{14} \mathrm{C}$ initial activity of calcareous deposits. Radiocarbon 34(3): 593-601.

Krajcar Bronić I, Horvatinčić N, Obelić B, Bistrović R. 1995. Radiocarbon intercomparison studies at the Rudjer Bošković Institute. Radiocarbon 37(2):80511.

Krajcar Bronić I, Horvatinčić N, Obelić B. 1998. Two decades of environmental isotope records in Croatia: Reconstruction of the past and prediction of future levels. Radiocarbon 40(1):399-416.

Krajcar Bronić I, Horvatinčić N, Barešić J, Obelić B. 2009. Measurement of ${ }^{14} \mathrm{C}$ activity by liquid scintillation counting. Applied Radiation and Isotopes 67(5): 800-4.

Krajcar Bronić I, Obelić B, Horvatinčić N, Barešić J, Sironić A, Minichreiter K. 2010. Radiocarbon application in environmental science and archaeology in Croatia. Nuclear Instruments and Methods in Physics Research A 619(1-3):491-6.

Lacković D, Šmida B, Horvatinčić N, Tibljaš D. 1999. Some geological observations in Slovačka Jama Cave $(-1268 \mathrm{~m})$ in Velebit Mountain, Croatia. Acta Carsologica 28:113-20.

Levin I, Kromer B. 2004. The tropospheric ${ }^{14} \mathrm{CO}_{2}$ level in mid-latitudes of the Northern Hemisphere (19592003). Radiocarbon 46(3):1261-72.

Mook WG, van der Plicht J. 1999. Reporting ${ }^{14} \mathrm{C}$ activities and concentrations. Radiocarbon 41(3):227-39.

Özkul M, Gökgöz A, Horvatinčić N. 2010. Depositional properties and geochemistry of Holocene perched springline tufa deposits and associated spring waters: a case study from the Denizli Province, western Turkey. In: Pedley HM, Rogerson M, editors. Tufa and Speleothems: Unravelling the Microbial and Physical Controls. London: The Geological Society Publishing House. p 245-62.

Pavlović G. 2001. Geokemijska, petrografska i mineraloška analiza sedri rijeka Zrmanje i Krupe [Geochemical, petrographical and mineralogical analysis of tufa from Zrmanja and Krupa rivers]. MSc thesis. Zagreb: Faculty of Natural Sciences. 63 p.

Pavlović G, Zupanič J, Prohi E, Tibljaš D. 2002. Impression of the biota associated with waterfalls and cas- 
cades from a Holocene tufa in the Zrmanja River Canyon, Croatia. Geologia Croatica 55(1):25-37.

Portner A, Obelić B, Krajcar Bronić I. 2010. ZAGRADA - the new Zagreb Radiocarbon Database. Radiocarbon 52(3):941-7.

Reimer PJ, Baillie MGL, Bard E, Bayliss A, Beck JW, Blackwell PG, Bronk Ramsey C, Buck CE, Burr GS, Edwards RL, Friedrich M, Grootes PM, Guilderson TP, Hajdas I, Heaton TJ, Hogg AG, Hughen KA, Kaiser KF, Kromer B, McCormac FG, Manning SW, Reimer RW, Richards DA, Southon JR, Talamo S, Turney CSM, van der Plicht J, Weyhenmeyer CE. 2009. IntCa109 and Marine09 radiocarbon age calibration curves, 0-50,000 years cal BP. Radiocarbon 51(4): $1111-50$.

Sironić A, Krajcar Bronić I, Horvatinčić N, Barešić J, Obelić B, Felja I. 2012. Status report on the Zagreb Radiocarbon Laboratory - AMS and LSC results of VIRI intercomparison samples. Nuclear Instruments and Methods in Physics Research B. In press. doi: 10.1016/j.nimb.2012.01.048.

Srdoč D, Breyer B, Sliepčević A. 1971. Rudjer Bošković Institute radiocarbon measurements I. Radiocarbon 13(1):135-40.

Srdoč D, Obelić B, Horvatinčić N, Sliepčević A. 1979. Measurement of the ${ }^{14} \mathrm{C}$ activity of the ANU sucrose secondary standard by means of the proportional counter technique. Radiocarbon 21(3):321-8.
Stuiver M, Polach HA. 1977. Discussion: reporting of ${ }^{14} \mathrm{C}$ data. Radiocarbon 19(3):355-63.

Surić M, Juračić M. 2010. Late Pleistocene-Holocene environmental changes - records from submerged speleothems along the Eastern Adriatic coast (Croatia). Geologia Croatica 63(2):155-9.

Surić M, Juračić M, Horvatinčić N. 2004. Comparison of ${ }^{14} \mathrm{C}$ and ${ }^{230} \mathrm{Th} /{ }^{234} \mathrm{U}$ dating of speleothems from submarine caves in the Adriatic Sea (Croatia). Acta Carsologica 33(2):239-48.

Surić M, Juračić M, Horvatinčić N, Krajcar Bronić I. 2005a. Late Pleistocene-Holocene sea-level rise and the pattern of coastal karst inundation: records from submerged speleothems along the Eastern Adriatic Coast (Croatia). Marine Geology 214(1-3):163-75.

Surić M, Horvatinčić N, Suckow A, Juračić M, Barešić J. 2005b. Isotope records in submarine speleothems from the Adriatic coast, Croatia. Bulletin de la Société géologique de France 176:363-73.

Surić M, Jalžić B, Petricioli D. 2007. Submerged speleothems - expect the unexpected. Examples from the Eastern Adriatic Coast (Croatia). Acta Carsologica 36(3):389-96.

Vokal B. 1999. The carbon transfer in Karst areas - an application to the study of environmental changes and paleoclimatic reconstruction [PhD thesis]. Nova Gorica: Polytechnics Nova Gorica. 